\title{
Biomarkers for immunotherapy in bladder cancer: a moving target
}

\author{
David H. Aggen ${ }^{*}$ and Charles G. Drake
}

\begin{abstract}
Treatment options for metastatic urothelial carcinoma (mUC) remained relative unchanged over the last 30 years with combination chemotherapy as the mainstay of treatment. Within the last year the landscape for mUC has seismically shifted following the approval of five therapies targeting the programmed cell death protein (PD-1)/programmed cell death ligand 1 (PD-L1) axis. Notably, the anti-PD-1 antibody pembrolizumab demonstrated improved OS relative to chemotherapy in a randomized phase III study for second line treatment of mUC; this level 1 evidence led to approval from the U.S. Food and Drug Administration (FDA). The PD-1 antibody nivolumab also demonstrated an overall survival benefit, in this case in comparison to historical controls. Similarly, antibodies targeting PD-L1 including atezolizumab, durvalumab, and avelumab have now received accelerated approval from the FDA as second line treatments for mUC, with durable response lasting more than 1 year in some patients. Some of these agents are approved in the first line setting as well - based on single-arm phase II studies atezolizumab and pembrolizumab received accelerated approval for first-line treatment of cisplatin ineligible patients. Despite these multiple approvals, the development of clinically useful biomarkers to determine the optimal treatment for patients remains somewhat elusive. In this review, we examine key clinical trial results with anti-PD1/PD-L1 antibodies and discuss progress towards developing novel biomarkers beyond PD-L1 expression.

Keywords: Bladder cancer, PD-1, Pd-L1, Immunotherapy, Atezolizumab, Nivolumab, Pembrolizumab, Avelumab, Durvalumab, Tumor mutation burden, Immune checkpoint inhibitor, Biomarker, Nanostring
\end{abstract}

\section{Background}

Approximately 79,000 new cases of bladder cancer are estimated in the United States in 2017, resulting in 16,870 deaths [1]. Worldwide, it is estimated that there will be $\sim 168,000$ deaths attributed to urothelial cancer in 2017 [2]. Although the majority of patients present with non-muscle invasive disease, approximately $30-40 \%$ of patients have muscle invasive disease at diagnosis with a worse prognosis. The five-year overall survival rate for all stages of urothelial cancer remains between 15 and 20\%. Despite advances in treatment and survival, over the past 30 years treatment regimens for metastatic urothelial carcinoma remained relatively unchanged until the emergence of programmed cell death protein (PD-1) and programmed death ligand 1 (PD-L1) immune checkpoint therapies [3-5]. Within the last 18 months five new immunotherapies have been approved for second-line treatment of metastatic urothelial

\footnotetext{
* Correspondence: dha2113@cumc.columbia.edu

New York-Presbyterian/Columbia University Medical Center, Hematology/ Oncology, 177 Fort Washington Avenue, 6GN-435, New York, NY 10032, USA
}

cancer (mUC) (Fig. 1). With this new armamentarium of treatment options focus has shifted toward developing novel biomarkers for treatment stratification. Here we review approved anti-PD-1 and anti-PD-L1 therapies and discuss future directions for combination immunotherapies. In addition, we highlight potential biomarkers to guide treatment decisions with particular attention to those that focus on the genetic level.

\section{Immunotherapy for metastatic Urothelial cancer}

The five immunotherapy agents that are FDA approved for the treatment of metastatic urothelial carcinoma all have similar objective response rates (ORR) - between 15 and $23 \%$ in unselected patients in the second line setting (Table 1). Atezolizumab, nivolumab, durvalumab, and avelumab were approved based on single-arm studies comparing median overall survival (OS) and ORR with historical controls. Pembrolizumab is the only therapy validated in a randomized, phase III trial. A brief review of the clinical trial data that led to each approval follows. 


\section{Clinical Trials}

\begin{tabular}{|c|c|c|c|}
\hline & $\begin{array}{l}\text { Checkmate } 032 \\
\text { (Phase I) } 2^{\text {nd }} \text { line } \\
\text { nivolumab }\end{array}$ & \multicolumn{2}{|c|}{$\begin{array}{l}\text { Keynote } 052 \\
\text { (Phase II) } 1^{\text {st }} \text { line } \\
\text { pembrolizumab }\end{array}$} \\
\hline $\begin{array}{l}\text { GO27831 } \\
\text { (Phase I) } \\
\text { atezolizumab }\end{array}$ & $\begin{array}{l}\text { IMvigor } 210 \text { Cohort } \\
2 \text { (Phase II) } 2^{\text {nd }} \text { line } \\
\text { atezolizumab }\end{array}$ & \multicolumn{2}{|c|}{$\begin{array}{l}\text { Checkmate } 275 \\
\text { (Phase II) } 2^{\text {nd }} \text { line } \\
\text { nivolumab }\end{array}$} \\
\hline 2015 & 2016 & \multicolumn{2}{|r|}{2017} \\
\hline \multicolumn{4}{|c|}{ FDA APPROVALS } \\
\hline & \multicolumn{2}{|c|}{$\begin{array}{l}\text { 5/18/16: atezolizumab } \\
\text { approved for } 2^{\text {nd }} \text { line } \\
\text { therapy of mUC }\end{array}$} & $\begin{array}{l}\text { 2/2/17: nivolumab } \\
\text { approved for } 2^{\text {nd }} \\
\text { line therapy of } \\
\text { mUC }\end{array}$ \\
\hline
\end{tabular}

\section{Atezolizumab}

Atezolizumab, a humanized anti-PD-L1 IgG1 antibody engineered to minimize binding to $\mathrm{Fc}$ receptors, was the first therapy granted approval by the FDA [6, 7]. Approval was based on the IMvigor 210 study, a singlearm phase II trial in which mUC patients received
MEDI4736-1108

(Phase I/II) $2^{\text {nd }}$ line

durvalumab

Keynote 012 (Phase IB)

$2^{\text {nd }}$ line pembrolizumab

IMvigor 210 Cohort 1:

(Phase II) $1^{\text {st }}$ line

atezolizumab
JAVELIN (Phase I) $2^{\text {nd }}$ line

avelumab

Keynote 045 (Phase III)

study of $2^{\text {nd }}$ line

pembrolizumab

\begin{tabular}{|c|c|}
\hline $\begin{array}{l}\text { 4/18/17: atezolizumab } \\
\text { accelerated approval for } \\
1^{\text {st }} \text { line (Cis-ineligible }\end{array}$ & $\begin{array}{l}\text { 5/8/17: avelumab } \\
\text { accelerated approval for } 2^{\text {nd }} \text { line } \\
\text { therapy of } m \cup C \text {. }\end{array}$ \\
\hline & 5/8/17: pembrolizumab \\
\hline $\begin{array}{l}\text { 5/1/17: durvalumab } \\
\text { accelerated approval for }\end{array}$ & $\begin{array}{l}\text { approval for } 2^{\text {nd }} \text { line therapy } \\
\text { of } \mathrm{mUC}\end{array}$ \\
\hline $2^{\text {nd }}$ line therapy of mUC & $\begin{array}{l}\text { 5/18/17: pembrolizumab } \\
\text { accelerated approval for } 1^{\text {st }} \text { line } \\
\text { (Cis-ineligible patients) }\end{array}$ \\
\hline
\end{tabular}

4/18/17: atezolizumab

$1^{\text {st }}$ line (Cis-ineligible patients

5/1/17: durvalumab accelerated approval fo (Cis-ineligible patients)

Table 1 Response rates and median overall survival with FDA approved anti-PD-1/PD-L1 blockade in metastatic urothelial carcinoma

\begin{tabular}{|c|c|c|c|c|c|c|c|c|}
\hline & Medication & Phase & \# Patients & ORR (\%) & PFS (m.) & OS (m.) & PD-L1 Response & Reference \\
\hline \multirow{7}{*}{$\begin{array}{l}\text { Metastatic } 2 \text { nd } \\
\text { Line Therapy }\end{array}$} & \multirow{2}{*}{ Atezolizumab } & I & 100 & 21.0 & - & 8 & - & Powles et al. [7] \\
\hline & & $\|$ & 310 & 15.0 & 2.1 & 7.9 & $\begin{array}{l}\text { PD-L1 on IC }>5 \% \text { associated with ORR, } \\
\text { testing not required for treatment }\end{array}$ & Rosenberg et al. [8] \\
\hline & \multirow[t]{2}{*}{ Pembrolizumab } & III & 270 & 21.6 & 2.1 & 10.3 & $\begin{array}{l}\text { PD-L1 TC and IC composite score }>10 \% \text {, } \\
\text { no difference in ORR or mOS }\end{array}$ & Bellmunt et al. [13] \\
\hline & & C & 272 & 6.7 & 3.3 & 7.4 & - & \\
\hline & Nivolumab & $\|$ & 270 & 19.6 & 2.0 & 8.74 & $\begin{array}{l}\text { PD-L1 on TC > 1\% not associated with } \\
\text { ORR but associated with OS }\end{array}$ & Sharma et al. [11] \\
\hline & Avelumab & $\mid \mathrm{lb} / \|$ & 241 & 17.6 & 1.6 & 7.0 & $\begin{array}{l}\text { PD-L1 on TC > 5\% associated with } \\
\text { improved ORR, no OS data as of yet }\end{array}$ & $\begin{array}{l}\text { Apolo et al. [22] } \\
\text { Patel et al. [23] }\end{array}$ \\
\hline & Durvalumab & $\mathrm{lb}$ & 191 & 17.8 & - & - & $\begin{array}{l}\text { Composite biomarker of PD-L1 > } 25 \% \text { on } \\
\text { TC or IC predicts response rates, } \\
\text { approved companion diagnostic }\end{array}$ & Massard et al. $[19,21]$ \\
\hline \multirow[t]{2}{*}{$\begin{array}{l}\text { Metastatic } \\
\text { 1st Line* }\end{array}$} & Atezolizumab & $\|$ & 100 & 23.0 & 2.7 & 15.9 & $\begin{array}{l}\text { PD-L1 on IC not associated with improved } \\
\text { ORR or mOS }\end{array}$ & Balar et al. [10] \\
\hline & Pembrolizumab & $\|$ & 370 & 29.0 & - & - & $\begin{array}{l}\text { PD-L1 TC and IC composite score with cutoff } \\
\text { of } 10 \% \text {, no difference noted in ORR }\end{array}$ & Balar et al. [16] \\
\hline
\end{tabular}

$1200 \mathrm{mg}$ of atezolizumab at 3 week intervals [8]. This trial had two cohorts; Cohort 2 of IMvigor enrolled patients who had disease progression during or following platinum-based chemotherapy or within 12 months of neoadjuvant or adjuvant therapy. Objective response rates in patients receiving atezolizumab were $14.8 \%$ of $10 \%$, no difference noted in ORR 
(CI 11.1-19.3) for the entire study population, with an ORR of $9.5 \%$ noted in patients with low PD-L1 immune cell (IC) expression $(<5 \%)$ and $26 \%$ in patients with high PD-L1 IC ( $>5 \%)$ immunohistochemistry (IHC). Of note, in this trial samples were assayed using the SP142 assay [9]. Based on a prespecified response rate of $10 \%$ in historical controls, the FDA-granted approval for atezolizumab for patients who progress after platinum-based therapy or who have progressed within 1 year of neoadjuvant or adjuvant therapy on a platinum containing regimen. The median overall survival in patients receiving second line atezolizumab was 7.9 months (CI 6.7-9.3 m.). At a median follow-up of 11.7 months, responses were ongoing in 38/45 responding patients (84\%), suggesting that a proportion of patients experienced sustained benefit.

The other cohort of this trial, Cohort 1, enrolled cisplatin-ineligible patients who were treated with first-line atezolizumab at an identical dosing schedule to cohort II [10]. The majority of patients in cohort 1 suffered from renal impairment that prohibited cisplatin based therapy (70\%). The ORR for that cohort of 123 patients was approximately $23 \%$, in comparison to a widely accepted historical control ORR of $10 \%$. The median overall survival for the entire cohort was 15.9 months (95\% CI: $10.4 \mathrm{~m}$. - not estimable) with $21 \%$ of the patient population remaining on therapy for more than 1 year. In surprising contrast to what was observed in cohort II, responses in cohort I appeared to be independent of PD-L1 status (ORR of $28 \%$ vs. $21 \%$ for $>5 \%$ PD-L1 IC expression and $<5 \%$ PD-L1 IC expression). Median overall survival was also independent of PD-L1 status $(12.3$ vs. 19.1 months for $>5 \%$ PD-L1 IC and $<5 \%$ PD-L1 IC expression. Based on the favorable ORR relative to historical controls the FDA approved atezolizumab for use in cisplatin-ineligible patients with metastatic urothelial carcinoma.

The most common adverse events (AE) noted with atezolizumab at the prespecified dose level in cohort I and cohort II were fatigue, diarrhea, and pruritis with rare instances of autoimmune phenomena commonly associated with PD-1 therapy including elevation in liver enzyme tests (3\%), pneumonitis (2\%) and hypothyroidism (7\%). Recently, the randomized phase III IMvigor 211 study evaluating atezolizumab in comparison to chemotherapy as second line treatment was announced to have failed to reach its primary endpoint of improved overall survival, independent of PD-L1 expression status. More extensive data on that trial were not available at the time of writing, but that unexpected outcome highlights the need for improved patient stratification beyond PD-L1 testing to select appropriate mUC patients for immunotherapy.

\section{Nivolumab}

Nivolumab, a fully-human IgG4 anti-PD1 antibody hinge-modified to improve half-life, received accelerated approval from the FDA for second line therapy in previously platinum treated mUC. This approval was based on data from the checkmate 275 trial, a single-arm phase II study that enrolled 270 patients to receive nivolumab at $3 \mathrm{mg} / \mathrm{kg}$ every 2 weeks [11]. The PD-L1 assay used in Checkmate 275 measured PD-L1 tumor cell expression using the 28-8 antibody (Dako PD-L1 IHC kit, Dako North America, Carpenteria, CA, USA) and differed from that in the IMvigor 210 cohort which measured immune cell PD-L1 expression using a different diagnostic antibody and staining protocol. Objective response rates for the entire population approached $19.6 \%$ in comparison to a widely accepted historical control objective response rate of $10 \%$. In contrast to what was observed with atezolizumab in cohort II of IMvigor 210 , responses appeared to be independent of PD-L1 tumor cell expression (objective response rates of $28.4 \%$, $23.8 \%$, and $16.1 \%$ were noted for tumor cell PD-L1 expression of $>5 \%$, $>1 \%$, or $<1 \%$ respectively). Conversely, median OS was 11.30 months for patients with $\geq 1 \%$ PD-L1 expression in comparison to 5.95 months with $\leq 1 \%$ PD-L1 expression. This compared favorably with historical controls from meta-analyses of second line chemotherapy that demonstrate a pooled median overall survival of 6.98 months [12]. The most common adverse events noted with nivolumab were grade 3 or 4 diarrhea, with $18 \%$ (48 of 270 patients) experiencing a grade 3 or $4 \mathrm{AE}$. There were 3 on-study deaths attributed to treatment; one case each of pneumonitis, acute respiratory failure, and cardiac compromise. Based on the overall response rate and relative safety, nivolumab was approved in February of 2017 for treatment of platinum refractory mUC.

\section{Pembrolizumab}

Pembrolizumab is a hinge-stabilized, humanized IgG4 anti-PD1 antibody that, like nivolumab, disrupts the engagement of PD-1 with its ligands PD-L1 and PD-L2. Of the FDA-approved antibodies blocking the PD-1/PD-L1 interaction, pembrolizumab is the only agent approved based on data from a randomized, phase III study [13]. Approval was granted by the FDA based on the openlabel Keynote-045 study which randomly assigned 542 patients who had recurred or progressed after platinumbased therapy to investigator's choice chemotherapy (paclitaxel, docetaxel, or vinflunine) or to pembrolizu$\mathrm{mab}$ at $200 \mathrm{mg}$ every 3 weeks. The median overall survival in the pembrolizumab arm was improved as compared to the chemotherapy arm (10.3 m., CI 8.011.8 vs. 7.4 m., CI $6.1-8.3$ m., $P=0.002$ ). Progression free survival was not improved versus chemotherapy; 
this has been observed in other phase III studies of PD-1 blocking agents $[14,15]$. The ORR for the pembrolizumab treated patients was significantly higher than in the chemotherapy group $(21.1 \%$ vs $11.4 \%, P=0.001)$. Consistent with what was observed in cohort I of IMvigor 210 with atezolizumab and Checkmate-275 with nivolumab, overall response rate was similar between groups with low and high PD-L1 expression as measured by tumor cell (TC) and immune cell (IC) PD-L1 expression using the Dako assay and the $22 \mathrm{C} 3$ antibody. I.E. the ORR was $21.1 \%$ in the total population versus $21.6 \%$ in the group with PD-L1 score of $>10 \%$. The lack of correlation between response rates and PD-L1 combined score again demonstrates an unmet need for biomarkers for treatment selection. The median overall survival of the PD-L1 high composite score group ( $>10 \%)$ was 8.0 months (CI 5.0-12.3) with pembrolizumab in comparison to 5.2 months (CI 4.0-7.4) in the chemotherapy group. While pembrolizumab clearly provides a survival benefit relative to chemotherapy, higher PD-L1 expression was not associated with increased survival relative to the entire pembrolizumab treatment group. Grade 3 or 4 adverse events were less frequent in the pembrolizumab group (15\% with pembrolizumab vs $49.4 \%$ in the chemotherapy arm). Similar to nivolumab the most frequently reported side effects were pruritus (19.5\%), fatigue (15.0\%), nausea (11.3\%), and diarrhea (10.1\%).

Pembrolizumab was also approved for use as first-line therapy in cisplatin ineligible patients in mUC based on early data from the phase II Keynote-052 study $[10,16]$. Overall survival data have not yet been reported. However, overall response rate was $27 \%$ for the entire study population with PFS and OS rates at 6 months of 31\% and $67 \%$ respectively. An exploratory end-point in this study was overall response rate in patients with a PD-L1 composite score of $>10 \%$; here the ORR approached $51 \%$ $(n=42)[16,17]$. In an effort generate a biomarker with a higher negative predictive value, an exploratory analysis was performed with an 18-gene expression signature designating a T-cell inflamed phenotype as assessed by Nanostring (described later) [17]. This companion assay has been validated in a small number of patients treated with pembrolizumab with metastatic melanoma, gastric cancer, and head and neck cancer [18] and showed an even better association with overall response than did PD-L1 expression.

\section{Durvalumab}

Durvalumab, an FcR binding deficient anti-PD-L1 antibody, was approved in May 2017 based on a single-arm phase I/II study evaluating 61 patients with platinum-treated advanced UC [19]. Patients were eligible if they had disease relapse within 1 year of neoadjuvant chemotherapy. The overall response rate for the entire cohort was $31.0 \%$. Response rates for patients with PD-L1 expressing tumor cells were $46.4 \%$ in comparison to $22 \%$ for PD-L1 negative tumors. Here PDL1 staining was performed using Ventana SP263 assay [9]. To select patients for durvalumab, an interesting composite biomarker defined PD-L1 'positivity' if either tumor cells (TC) or immune cells (IC) demonstrated $\geq 25 \%$ staining by IHC [20]. In contrast, a patient was considered PD-L1 negative if both tumor cell and immune cell expressed $\leq 25 \%$ PD-L1. Utilizing this new composite biomarker, patients with negative PD-L1 expression in tumor cells and immune cells had an ORR of $0 \%$ (0 of 14) in comparison to an ORR of $46 \%$ for patients positive for PD-L1 in either compartment. A recent follow-up analysis reporting on 191 patients treated with durvalumab reported an ORR of $17.8 \%$ with an enrichment in response rates for PD-L1 high patients (ORR $27.6 \%$ vs. 5.1\%) [21]. The FDA approved durvalumab along with a companion assay in May of 2017 (Ventana SP263).

\section{Avelumab}

The single-arm JAVELIN phase I study evaluated avelumab, an IgG1 anti-PD-L1 antibody that blocks the interaction between PD-1 and PD-L1 but not PD-1 and PD-L2. In an initial phase Ib study of unselected patients with platinum refractory mUC the ORR was $18.2 \%$ with a reported median OS of 13.7 months [22]. All patients in the initial phase Ib cohort of 44 patients developed an adverse event, with infusion reactions noted in approximately $20 \%$ of patients. At a primary end-point of 12-weeks there was a trend towards increased survival in high PD-L1 expressing patients, with ORR of $53.8 \%$ vs $9.0 \%$ in PD-L1 tumor cell high relative to low expressing tumors (cutoff 5\%, Dako PD-L1 IHC kit, Dako North America, Carpenteria, CA, USA, Merck 73-10 monoclonal antibody) [9]. Recently, a pooled analysis of an additional cohort of 241 patients with platinum refractory UC demonstrated a confirmed ORR of $17.6 \%$ [23]. The median overall survival for the pooled cohort approached 7.0 months (CI: 5.6-11.1). Using a similar cutoff of 5\% PDL1 tumor cell positivity, the ORR were $25 \%$ versus $14.7 \%$ for high and low PD-L1 expression respectively, with $6 \%$ of patients having severe AEs. The most common AEs noted with avelumab in more than $10 \%$ of patients included infusions reactions (22.8\%) and fatigue (12.0\%), with $11.6 \%$ of patients experiencing an autoimmune adverse event and 1 treatment related death attributed to pneumonitis. Based on comparison to historical controls, the FDA approved avelumab for 2nd line treatment of platinum-refractory locally advanced or metastatic UC.

\section{Combination immunotherapy trials}

Extrapolating from studies in melanoma [24] and NSCLC [25], multiple trials are now aimed at evaluating combination PD-1/CTLA-4 blockade. Preliminary data from the randomized phase I/II Checkmate-032 trial showed safety of the combination at 2 dose levels resulting in ORR of 
$38.5 \%$ and $26 \%$ at higher and lower doses of ipilimumab respectively ( $3 \mathrm{mg} / \mathrm{kg}$ ipilimumab and $1 \mathrm{mg} / \mathrm{kg}$ nivolumab relative to $1 \mathrm{mg} / \mathrm{kg}$ ipilimumab and $3 \mathrm{mg} / \mathrm{kg}$ nivolumab) [26]. Given the improved overall response rate of 38.5\% with nivolumab $(1 \mathrm{mg} / \mathrm{kg})$ and ipilimumab $(3 \mathrm{mg} / \mathrm{kg})$ relative to the $26 \%$ ORR observed with nivolumab monotherapy, a phase III study is planned to assess efficacy of the combination therapy (Checkmate-901, NCT03036098). In patients who have progressed on nivolumab, a small cohort of patients has been challenged with ipilimumab/nivolumab with response rates noted in $10 \%$ of patients [27]. Similarly, a phase III trial with targeted enrollment of 525 patients is accruing using combination durvalumab and tremelimumab in comparison to standard of care first-line chemotherapy [28].

A number of on-going trials are evaluating novel targets in combination with PD-1 therapy, including traditional chemotherapy [29], intra-vesical BCG, IDO inhibitors such as epacadostat [30], CD27 [31], CD137, OX-40 [32], and CSF1-R [33] (Tables 2 and 3). Recent phase I efforts explored the safety of combination nivolumab and ipilimumab with other approved tyrosine kinase inhibitors; data demonstrating the safety of combined nivolumab, ipilimumab, and cabozantinib was recently presented across genitourinary malignancies [34]. As the number of combination trials continue to expand, pre-clinical and translational validation of these targets will be essential to select combinations with the highest likelihood of efficacy.

With the continued success of PD-1 targeted therapies in the metastatic setting a number of studies are evaluating immune checkpoint blockade in BCG-refractory non-muscle invasive bladder cancer. Early phase clinical trials evaluating BCG in combination with both pembrolizumab [35] (NCT02324582, NCT02808143) and atezolizumab [36] (NCT02792192) are currently accruing. It remains in open question if the potential autoimmune side effects related to immune checkpoint blockade will offset the potential benefits of PD-1 blockade in nonmuscle invasive disease.

Table 2 On-going combination immunotherapy trials in urothelial cancer

\begin{tabular}{|c|c|c|c|c|}
\hline Therapy & Number & Phase & Trial ID & Est. Completion \\
\hline Nivolumab +/- Ipilimumab (CheckMate-032) & $\begin{array}{l}1150 \\
70\end{array}$ & $\begin{array}{l}1 / \| \\
\|\end{array}$ & $\begin{array}{l}\text { NCT01928394 } \\
\text { NCT02553642 }\end{array}$ & $\begin{array}{l}\text { December } 2018 \\
\text { September } 2018\end{array}$ \\
\hline Atezolizumab + MOXR0916 (anti-OX40) +/- Bevacizumab & 762 & I & NCT02410512 & August 2018 \\
\hline CPI-444 + Atezolizumab & 534 & $\mathrm{l} / \mathrm{lb}$ & NCT02655822 & June 2018 \\
\hline Pembrolizumab + PLX3397 (CSF1R) & 400 & $|/| \mid$ & NCT02452424 & July 2019 \\
\hline BMS-986106 (anti-LAG3) +/- Nivolumab & 360 & $|/| \mid$ & NCT01968109 & October 2019 \\
\hline MK-7684 +/- Pembrolizumab & 336 & । & NCT02964013 & March 2020 \\
\hline GSK3174998 (anti-OX40) +/- Pembrolizumab & 264 & । & NCT02528357 & January 2020 \\
\hline Pembrolizuamb + Lenvatinib & 250 & $\mid \mathrm{b} / \|$ & NCT02501096 & January 2018 \\
\hline Durvalumab + Epacadostat & 185 & $|/| \mid$ & NCT02318277 & January 2018 \\
\hline Pembrolizumab + Ramucirumab & 155 & 1 & NCT02443324 & June 2018 \\
\hline Nivolumab + Cabozantinib +/- Ipilimumab & 135 & I & NCT02496208 & December 2017 \\
\hline Atezolizumab + Epacadostat & 118 & I & NCT02298153 & November 2020 \\
\hline $\begin{array}{l}\text { Durvalumab + (Olaparib or Vistusertib or } \\
\text { AZD1775 or AZD4547) }\end{array}$ & 110 & l & NCT02546661 & March 2019 \\
\hline Durvalumab + Tremelimumab + polyICLC (TLR3 agonist) & 102 & $|/| \mid$ & NCT02643303 & August 2022 \\
\hline Pembrolizuamb +/- Acalabrutinib & 75 & $\|$ & NCT02351739 & Summer 2017 \\
\hline Tremelimumab +/- Durvalumab & 64 & $\|$ & NCT02527434 & October 2018 \\
\hline Ipilimumab + Enoblituzumab (anti-B7-H3) & 59 & । & NCT02381314 & March 2018 \\
\hline Atezolizumab + B-701 (FGFR3 inhibitor) & 48 & $\mathrm{lb}$ & NCT03123055 & April 2017 \\
\hline Pembrolizumab + Vorinostat & 42 & 1 & NCT02619253 & May 2018 \\
\hline Pembrolizumab + Docetaxel or Gemcitabine & 38 & । & NCT02437370 & December 2019 \\
\hline Nivolumab + Enadenotucirev (oncolytic virus) & 30 & I & NCT02636036 & June 2018 \\
\hline Avelumab + NHS-IL-12 & 30 & । & NCT02994953 & April 2018 \\
\hline Pembrolizumab + Paclitaxel & 27 & $\|$ & NCT02581982 & March 2019 \\
\hline Nivolumab + IFN- $\gamma$ & 15 & I & NCT02614456 & December 2017 \\
\hline
\end{tabular}


Table 3 On-going studies evaluating first-line therapies for metastatic urothelial cancer

\begin{tabular}{|c|c|c|c|c|c|}
\hline Therapy & Number & Phase & Primary Endpoint & Trial ID & $\begin{array}{l}\text { Estimated Completion } \\
\text { Date }\end{array}$ \\
\hline $\begin{array}{l}\text { Atezolizumab + Gemcitabine/Carboplatin vs. Gemcitabine/Carboplatin } \\
\text { (IMvigor 130) }\end{array}$ & 1200 & III & $\mathrm{PFS} / \mathrm{OS}$ & NCT02807636 & July 2020 \\
\hline Pembrolizumab +/- Platinum vs Gemcitabine/Platinum (Keynote 361) & 990 & III & $\mathrm{PFS} / \mathrm{OS}$ & NCT02853305 & March 2020 \\
\hline Durvalumab +/- Tremelimumab vs. Gemcitabine/Carboplatin (1:1:1) & 525 & III & $\mathrm{PFS} / \mathrm{OS}$ & NCT02516241 & July 2019 \\
\hline Pembrolizumab + CVA21 (Coxsackievirus A21) & 90 & । & Safety & NCT02043665 & August 2019 \\
\hline Nivolumab + NEO-PV-1 (personalized peptide vaccine) & 90 & $\mathrm{lb}$ & Safety & NCT02897765 & December 2020 \\
\hline Pembrolizumab + sEphB4-HSA & 60 & $\|$ & OS & NCT02717156 & November 2020 \\
\hline Gemcitabine/Cisplatin +/- Ipilimumab (Active, not accruing) & 36 & $\|$ & Safety/ORR & NCT01524991 & November 2017 \\
\hline $\begin{array}{l}\text { Atezolizumab +/- Gemcitabine Cisplatin } \\
\text { (First line metastatic or MIBC) }\end{array}$ & 30 & $|/| \mid$ & Safety & NCT02989584 & December 2020 \\
\hline
\end{tabular}

\section{Biomarkers for PD-1/PD-L1 blockade in mUC PD-L1 Expresssion}

Expression of PD-L1 has been noted in between 20 and $30 \%$ of urothelial cancer specimens [37, 38]. More important, PD-L1 expression as measured by IHC in bladder tumors is associated with increased pathologic stage at resection and increased all-cause mortality, suggesting that high levels may indicate more aggressive disease $[37,39]$. These data show that PD-L1 expression is prognostic in terms of outcome, a factor that needs to be taken into account when considering its predictive power in the context of PD-1 / PD-L1 targeted treatment. In urothelial cancer, phase II and phase III studies evaluated endpoints related to PD-L1 expression. PD-L1 staining assays and clinical results varied significantly across clinical trials in mUC highlighting the difficulties in using PD-L1 as a single biomarker. Data range from a strong association with overall responses using a composite biomarker that is required for patient selection (durvalumab [19, 21]) to no association as was noted in IMVigor Cohort 2 (atezolizumab 2nd line [8]), Keynote-045 (pembrolizumab [13]), and Checkmate-275 (nivolumab [11]).

One potential reason for these discrepancies is the use of 4 distinct assays for PD-L1 IHC scoring. For instance, pembrolizumab and nivolumab clinical trials use the Dako assay with the 22C3 and 28-8 antibody clones respectively. In contrast, durvalumab and atezolizumab use the Ventana assay and SP26 and SP142 antibody clones respectively [40]. In the setting of NSCLC, the four available anti-PD-L1 diagnostic assays have been compared with a greater degree of variability noted in PD-L1 IHC on immune cells [9]. In contrast, IHC of PD-L1 on tumor cells was comparable between the 22C3, 28-8, SP263 assays, whereas the SP142 assays showed significantly fewer PD-L1 positive tumor cells [14]. Another comparison of the 22C3, 28-8, SP26, and SP142 antibodies in 90 NSCLC specimens confirmed that SP-142 detected significantly lower mean PD-L1 TC and IC expression - again illustrating the challenges of comparing PD-L1 expression between assays [41]. In addition to inter-assay variability the scoring compartment differs for each specific therapy. Studies with pembrolizumab and nivolumab use PD-L1 tumor cell (TC) expression, whereas the IMVigor trials with atezolizumab use PD-L1 immune cell (IC) expression. In trials with durvalumab in $\mathrm{mUC}$, a composite end-point was used as described above with $25 \%$ TC or IC expression delineated as high PD-L1 expression [20]. Aside from these technical challenges the PD-L1 tumor and immune cell status may not reflect the meaningful PD-1/PD-L1 interactions necessary for predicting a $\mathrm{T}$ cell response. Intratumoral heterogeneity may further limit the utility of PD-L1 IHC due to incomplete sampling and differential expression of PD-L1 not adequately represented in the biopsy specimen. Analysis of PD-L1 expression in whole tissue sections of NSCLC noted a discordance approaching $25 \%$ based on the section selected for IHC [42]. Finally, PD-L1 tumor status fails to account for PD-1/PD-L1 interactions that may be occurring in the tumor draining lymph nodes. Perhaps most importantly, PD-L1 as a tumor marker is dynamic over time and space and a single biopsy may not reflect the local effects on the cytokine milieu or the immune landscape in its entirety. Taken together it appears unlikely that PD-L1 as a stand-alone biomarker will achieve sufficient positive or negative predictive value to effectively guide treatment decisions.

\section{Clustering by TCGA subtype}

Exploratory analyses in several trials retrospectively correlated The Cancer Genome Atlas (TCGA) urothelial cancer subtype with response to PD-1 / PD-L1 directed immunotherpy [43]. In cohort II (post chemotherapy) of the IMvigor210 study, TCGA classification was used to group patients into luminal $(n=73)$ or basal $(n=122)$ subtypes. Enrichment in PD-L1 immune cell expression was noted in the basal subtype (60\% vs $23 \%)$, while tumor cell PD-L1 expression was noted almost 
exclusively in basal subtypes (39\% vs $4 \%$ ). Responses to atezolizumab were documented in all subtypes with a statistically higher response rate noted in luminal cluster II subtype (ORR $=34 \%, p=0.0017$ ) relative to luminal cluster I, basal cluster I, and basal cluster II (ORR 10\%, $16 \%$, and $20 \%$ respectively). A similar trend was noted in Cohort I of IMvigor with atezolizumab with the highest percentage of responses noted in the luminal cluster II group $(n=11 / 37,7$ partial responses and 4 complete responses). The TCGA subtypes were also an exploratory endpoint in the phase II Checkmate-275 study of nivolumab; by contrast here basal I subtype tumors represented the highest proportion of responders $(7 / 23$, ORR $30 \%$ for basal I). Luminal cluster II tumors treated with nivolumab had an overall response rate of $\sim 25 \%$. The reasons for these discrepancies in the mUC subtype most likely to respond might be related to tissue source. Both cohorts of IMVigor210 and Checkmate-275 allowed biopsy specimens from primary tumor, lymph nodes, or metastatic lesions for TCGA subtyping which may lead to inappropriate tumor classification. Second, the criteria for molecular subtyping differs in each study, highlighting a challenge in standardizing TCGA classification. Taken together these results are consistent with the notion that TCGA subtype is not likely to prove a strong predictive biomarker, especially across agents.

\section{Tumor mutational burden/Neoantigen burden}

In both melanoma [44] and NSCLC [45], mutational load, as well as the number of predicted neoantigens is associated with a greater likelihood of durable responses to immune checkpoint blockade. In fact, those retrospective data suggest that mutational load may potentially predict response more robustly than PD-L1 IHC, presence of tumor infiltrating lymphocytes, or clinical variables [45]. While neoantigens have been identified by exome sequencing and validated with $\mathrm{T}$ cell activation assays - relatively few shared neoantigens have been identified and most neoantigens are likely to be patient specific [46-48]. As a consequence, a high nonsynonomous mutation burden may correlate to an increased number of neoantigens and data from focused exome sequencing has demonstrated a correlation between tumor mutation burden (TMB) and response rates to immunotherapy. An exploratory subgroup analysis of IMvigor210 Cohort II evaluating 315 cancer related genes showed a significant increase in mutation load in responding relative to non-responding patients (12.4 per megabase vs. 6.4 per megabase, $P<0.0001$ ) [8]. Smoking status and TCGA subtype did not correlate with mutational burden in a subset of 150 patients from IMvigor Cohort II, suggesting that TMB may be a more reliable predictor of response to PD-L1 blockade in urothelial cancer. Similarly, in cohort I of IMvigor 210,
119 tumor specimens were analyzed to determine tumor mutation load [10]. There was a clear correlation toward improved overall survival in the highest quartile of TMB ( $>16$ to $<62.2$ mutations per megabase) relative to quartiles 1-3, with a Kaplan-Meier estimated survival probability approaching $75 \%$ at 1 year (Fig. 2a). The effect in improvement in overall survival was independent of TCGA subtype, with responses noted in all four TCGA subgroups (Fig. 2b). Of note, these data appear to suggest the possibility of a threshold effect, with patients in the lowest 3 quartiles appearing similar, whilst the top quartile seems to have an increased likelihood of responding and an overall survival benefit. The use of mutation burden to predict responses to immunotherapy was also highlighted in an exploratory analysis from the prospective checkmate-026 study evaluating nivolumab in first-line therapy for NSCLC. Checkmate-026 randomized patients with metastatic NSCLC and $>1 \%$ PDL1 expression to platinum-based chemotherapy or nivolumab [49, 50]. An exploratory secondary end-point assessed progression free survival (PFS) based on tumor mutation burden for $\sim 50 \%$ of the study population who had tumor tissue available [51]. Patients with low or medium mutation burden status receiving nivolumab had inferior PFS relative to patients receiving standard of care chemotherapy (Fig. 2c and d). In contrast, patients with high levels of mutational burden responded favorably to nivolumab with improved median PFS, suggesting that high tumor mutation burden may predict responses to immunotherapy. Again, a threshold effect seemed to be present, with the lowest $2 / 3 \mathrm{rd}^{\mathrm{s}}$ showing a similar RR and the top $1 / 3^{\text {rd }}$ deriving a progression free survival benefit.

Other studies used retrospective data to evaluate the relationship between the number of non-synonomous mutations and immunotherapy responses. Data in NSCLC using targeted exome sequencing of cancer specific genes identified an association between high mutation burden and durable overall response [52]. A composite score of PD-L1 status $>1 \%$ and tumor mutation burden above the median correlated with response, with $60 \%$ of patients with high PD-L1 and high TMB deriving durable clinical responses. In a separate review of previously published data evaluating responses in melanoma and NSCLC, a threshold of 192 non-synonomous mutations (nSM) was hypothesized beyond which the the response rate to immunotherapy plateaus [53]. Using a cutoff of $192 \mathrm{nSM}$ a $74 \%$ sensitivity and $59.3 \%$ specificity was identified to discriminate a potential benefit. The negative predictive value of TMB, however, is unclear, as responses were observed in some patients with low mutation burden.

Significant challenges confront the use of TMB as a predictive biomarker for immunotherapy. First is the 

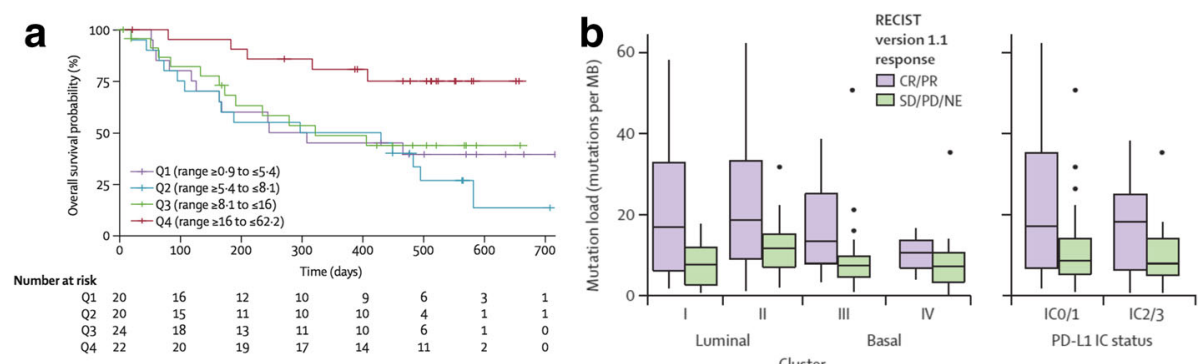

C

d

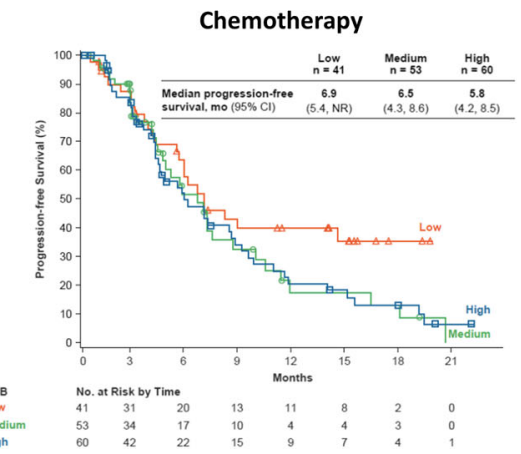

Fig. 2 Tumor mutation burden as a biomarker for anti-PD-1/PD-L1 therapy. a Kaplan-Meier estimate of overall survival according to estimated mutational burden by quartiles in mUC patients treated with atezolizumab in IMVigor 210 - Cohort I. Range estimates next to each qauartile indicated number of mutations per megabase for each quartile. $\mathbf{b}$ Quantification of mutation burden across TCGA subtypes and PD-L1 immune cell IHC status and correlation with disease status. $\mathbf{c}$ and $\mathbf{d}$ Progression-free survival based on tertile of tumor mutation burden from Checkmate 026, a randomized study of nivolumab (c) compared to standard of care chemotherapy (d). A and B reprinted from The Lancet, Vol. 389, Balar et al. "Atezolizumab as first-line treatment in cisplatin-ineligible patients with locally advanced and metastatic urothelial carcinoma: a single-arm, multicenter, phase 2 trial, p. 73, 2017 with permission from Elsevier [10]. C and D reproduced with permission from Carbone, D. et al. NEJM. 2017., [48]

challenge of unifying and standardizing the definition of mutation burden. For instance, some assays standardize for the size of the genome covered by targeted sequencing on a per megabase level. Others report based on absolute mutational burden which may fail to represent the true tumor mutation burden relative to the depth of sequencing performed. Second, gene fusions, truncations, and translocations may not be adequately covered by targeted sequencing panels and the value of these genetic events relative to single nucleotide variants in predicting response to immunotherapy remains to be determined. Third, germline variants may not be silenced by informatics techniques that filter common germline single nucleotide polymorphisms. As a consequence, uncommon germline variants may artificially increase the calculated tumor mutation burden, which highlights the need to improve standardization between tumor mutation burden assays. The somatic mutation burden is also likely to change dependent on other variables through the treatment course such as prior chemotherapy treatment and a biopsy at a single time point may not adequately reflect the relative antigenicity of the tumor. Despite these limitations there is now strong evidence that TMB correlates with durable responses to PD-1 blockade in multiple tumor types and with further standardization TMB will likely be a reliable surrogate to predict immunotherapy response.

Other surrogate measures of mutation burden such as chronic carcinogen exposure (eg. tobacco, ultraviolet light, aniline dye), defects in DNA repair mechanisms such as microsatellite instability/mismatch repair defects, and POLE mutations have emerged as potentially useful clinical biomarkers [45, 54]. Based on this notion and data demonstrating an overall response rate of $39.6 \%$ with pembrolizumab in microsatellite instability (MSI) high and mismatch repair deficient malignancies, pembrolizumab has been approved for patients with these gene defects solid after progression on prior treatments prompting investigation in a phase III setting [55-57]. This landmark FDA indication represents the first approval based on a tumor biomarker independent of tumor cell origin. Interestingly, certain mutational variants may portend a lack of benefit with PD-1 therapy, such as individual mutations in EGFR and STK11 that are associated with a lack of benefit in NSCLC and lung adenocarcinoma [52, 58].

Composite variables integrating PD-L1 expression, TCR sequencing/TCR clonotypes, epigenetic analysis, and tumor mutation burden may delineate characteristics 
that predict responses to immunotherapy due to inherent advantages and disadvantages of each biomarker as a stand alone test (Fig. 3). These individual modalities are reviewed extensively elsewhere [59-61].

There are now numerous examples across solid tumor types including head and neck squamous cell cancer, NSCLC, melanoma, and urothelial cancer exploring correlation between composite markers and response to anti-PD1 [62, 63]. In mUC, whole exome sequencing, RNA sequencing, and $\mathrm{T}$ cell receptor sequencing were performed on pre and post-treatment biopsies of patients treated with atezolizumab to evaluate predictors of durable disease control [64]. In a small cohort of 24 patients a combination biomarker of elevated PD-L1 immune cell staining and high TCR clonality pretreatment were associated with poor clinical outcomes. In the same cohort mutation load was evaluated with different methodologies including missense mutational load, predicted neoantigen load, and expressed neoantigen load. All of these additional methodologies failed to demonstrate any association with control of disease for 6 months, highlighting the need to standardize these assays and develop improved composite biomarkers, which may ultimately hinge on the use of gene expression signatures.

Ongoing efforts evaluating combined measurements of mutational load with gene expression signatures show promise. Gene expression profiling performed in longitudinal tumor biopsies showed dynamic changes in multiple genes after the initiation of PD-1 therapy [65]. As these immune signatures are refined there is the potential that on-treatment biopsies may guide treatment decisions based on immune cell gene expression rather than based on imaging. To date, exploratory subanalysis have looked at larger, less well-validated panels in an attempt to better define an optimal immune signature using high numbers of reproducible gene transcripts.

\section{Multiparameter immune gene expression profiling}

An inherent difficulty in using PD-L1 status as a predictive biomarker is that subjective scoring of IHC sections provides information regarding only a single factor in the tumor microenvironment, and doesn't take into account other features that might more accurately segregate "hot" from "cold" tumors $[66,67]$. In that regard, targeted gene expression panels may have the ability to quantify specific RNA expression profiles from formalinfixed paraffin embedded (FFPE) biopsy and more comprehensively delineate an inflamed tumor microenvironment. An advantage of immune gene expression profiling is that RNA can be quantified from multiple cell types within a specimen which might be more fully representative of the tumor microenvironment (described in Fig. 4a). Immune expression profiling has the potential to accurately determine the inflammatory status of a tumor by quantifying chemokines, cytokines, and cell surface proteins that may better approximate a "hot" tumor than PD-L1 expression alone.

In the Checkmate 275 study with nivolumab in $\mathrm{mUC}$, a 25-gene interferon-gamma (IFN- $\gamma$ ) signature derived from

\begin{tabular}{|c|c|c|}
\hline & Advantages & Disadvantages \\
\hline $\begin{array}{c}\text { PD-L1 } \\
\text { Immunohistochemistry }\end{array}$ & $\begin{array}{l}\text { - Most well-characterized biomarker to date } \\
\text { - Rapid turn around time from biopsy } \\
\text { - } \quad \text { Relatively inexpensive }\end{array}$ & $\begin{array}{l}\text { Discordant results across studies } \\
\text { - Poor negative predictive value: responses seen in PD-L1 negative tumors } \\
\text { - Unctiple antibodies in use to detect PD-L1 } \\
\text { microenvironment } \\
\text { - Biomarker is dynamic over time and does not reflect PD-1/PD-L1 interactions in } \\
\text { tumor draining lymph nodes } \\
\text { - Does not assess status of the immune microenvironment }\end{array}$ \\
\hline TCGA Subtyping & $\begin{array}{l}\text { - Evidence of increased immunotherapy response in } \\
\text { luminal cluster II subtypes with atezolizumab } \\
\text { Basal cluster I subtype demonstrated increased ORR } \\
\text { with nivolumab therapy } \\
\text { Distinct classifications based on tumor gene } \\
\text { signatures (i.e. few patients with gene signatures } \\
\text { between groups) }\end{array}$ & $\begin{array}{l}\text { - Multiple gene cluster assays used, difficult to standardize } \\
\text { TCGA subtyping in patients treated with immunotherapy is limited to small } \\
\text { numbers in each cohort (<60 patients in IMVigor study) } \\
\text { - May require deep sequencing to appropriately identify the TCGA subtype } \\
\text { - Responses are achieved in all } 4 \text { TCGA clusters, suggesting a low negative } \\
\text { predictive value } \\
\text { - Does not assess status of the immune microenvironment }\end{array}$ \\
\hline $\begin{array}{c}\text { Tumor Mutational } \\
\text { Burden }\end{array}$ & $\begin{array}{l}\text { - Clear examples of durable responses ( }>6 \text { months) } \\
\text { in patients with high mutation burden } \\
\text { Correlation demonstrated in subgroup analyses } \\
\text { between tumor mutation burden and overall } \\
\text { response rates with atezolizumab and } \\
\text { pembrolizumab }\end{array}$ & $\begin{array}{l}\text { - Difficult to standardize between sequencing assays } \\
\text { - Relative weight of SNPs and translocations not yet elucidated } \\
\text { undefinship between tumor mutation burden and neoantigen burden is still } \\
\text { - Depth of sequencing required to predict responders vs nonresponders } \\
\text { undetermined } \\
\text { - Evolution of tumor over time may change the relative mutation burden } \\
\text { - Does not assess status of the immune microenvironment }\end{array}$ \\
\hline $\begin{array}{l}\text { Immune Cell Gene } \\
\text { Expression Profiling }\end{array}$ & $\begin{array}{l}\text { - Higher reproducibility relative to PD-L1 IHC to } \\
\text { predict immunotherapy responses } \\
\text { - Only biomarker assessing immune cell status rather } \\
\text { than tumor characteristics } \\
\text { - Correlated with response to therapy in subgroup } \\
\text { analyses of nivolumab and pembrolizumab trials }\end{array}$ & $\begin{array}{l}\text { - No standardized commercially available gene panel as of yet. Multiple gene } \\
\text { panels currently available (T-cell panel, combined T-cell tumor cell panel, IFN- } \gamma \\
\text { specific) } \\
\text { - Insufficient negative predictive value: responders seen in all groups } \\
\text { - Cost }\end{array}$ \\
\hline
\end{tabular}



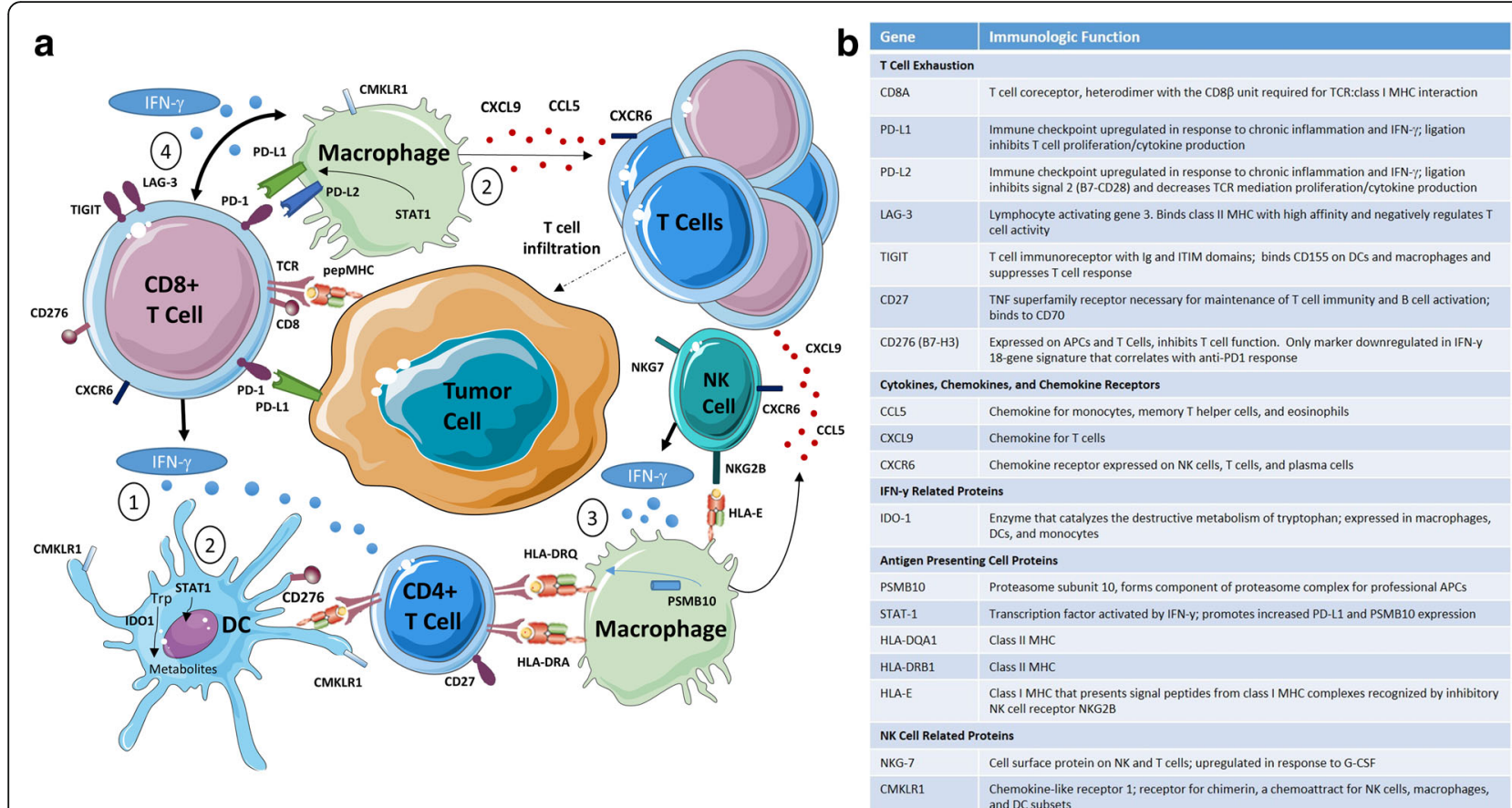

Fig. 4 Components of the tumor inflammatory signature as assessed by immune cell gene expression profiling. a Complex interplay of chemokines and cytokines classify the inflammatory state of the tumor microenvironment. Interferon-g (IFN-g) released by activated T cells and NK cells activates STAT1, IDO-1 (indolamine oxygenase I) and CMKLR1 in dendritic cells and macrophages (1). STAT-1 mediated signaling and additional pathways produce the chemokines CCL5 and CXCL9 (2). This recruits additional T cells into the tumor microenviroment through CXCR6. IFN-g stimulates the expression of HLA molecules and proteasome components including PSMB10 (3). Finally, IFN-g upregulates a number of immune checkpoint molecules including PD-L1, PD-L2, TIGIT, LAG-3, and B7-H3 on T cells (4). b Components of 18-gene immune signature under evaluation in prospective trials with pembrolizumab

crude extract (EdgeSeq, HTG Molecular Diagnostics Tucson, AZ, USA) was used to assess 177 tumor samples from pretreatment biopsies. Higher values in the IFN- $\gamma$ gene signature were correlated with response to nivolumab relative to low value IFN- $\gamma$ expression score $(p=0.0003$, CR or PR in $20 / 59$ patients with high IFN- $\gamma$ signature relative to CR or PR in 19/118 patients with medium or low IFN- $\gamma$ signature). Similar gene expression analysis performed with a chemokine panel showed enrichment in responses from individuals with high expression of CXCL9 and CXCL10 demonstrating the potential to use gene expression profiling as a biomarker. Similar to TMB measurements, the negative predictive value of this gene panel remains problematic as some responses were noted in some patients with a non-inflamed cytokine signature.

Next generation RNA expression technologies allow for immune profiling of greater than 700 genes from isolated RNA. For instance, NanoString nCounter (NanoString Technologies, Seattle, WA) uses 6-color bar codes to identify specific RNA sequencing without gene amplification as is required with traditional RNA sequencing or qPCR technologies. Using a small subset of 19 melanoma patients from Keynote-001, 680 different genes were profiled by Nanostring. A subset of 18 specific genes including interferon-gamma signaling (IDO1 and STAT1), antigen presentation (HLA-DRB1, HLADQA1, HLA-E), NK T cell signaling (NKG7, CMKLR1), and additional immunomodulatory proteins (Fig. 4b) were tested in a larger cohort of melanoma patients treated with pembrolizumab in Keynote-001. Validation of the test set in a cohort of patients with head and neck and gastric cancers from the KEYNOTE-028 trial showed a correlation with response to PD-1 therapy, with a deviation of $<5 \%$ in anti-PD- 1 response predictor score $[68,69]$. This 18-gene panel has now been validated using tumor specimens from patients across 9 tumor types in 220 patients treated with PD-1 therapy [18] and is currently being evaluated prospectively in 3 ongoing Phase III trials with pembrolizumab (NCT02628067 [70], NCT02559687 [71], and NCT02564263 [72]). The utility of nanostring-based gene expression signatures to predict response to immunotherapy hinges on the results of these pivotal phase III prospective studies - but if successful these data may ultimately guide treatment decisions in mUC and other immunotherapy responsive tumors.

\section{Conclusions}

The FDA approvals of atezolizumab, nivolumab, pembrolizumab, avelumab, and durvalumab represent a major paradigm shift in treating mUC. The recent 
results of the Phase III IMvigor 211 study, however, suggest the possibility that not all PD-1/PD-L1 reagents will have comparable efficacy. Standardized, reproducible biomarkers (potentially in composite) are needed to accurately guide treatment decisions as no single test has as of yet demonstrated reproducibility to predict responders to immunotherapy. This is of particular importance as there are potentially subgroups of patients with low mutation burden who may respond more favorably to chemotherapy as was noted in Checkmate 026. Although composite biomarkers are of interest, the next generation of predictive biomarkers for immunotherapy might involve either an assessment of tumor mutational burden (TMB) or a targeted gene expression profile with particular attention to $\mathrm{T}$ cell gene signatures; these ongoing studies are of critical importance in optimizing precision immunotherapy for patients with metastatic urothelial cancer.

\begin{abstract}
Abbreviations
AE: Adverse event; Cl: Confidence interval; FDA: Food and Drug Administration; IC: Immune cell; IHC: Immunohistochemistry; MSI: Microsatellite instability; mUC: Metastatic urothelial cancer; NSM: Non synonomous mutation; ORR: Objective response rate; OS: Overall survival; PD-1: Programmed cell death protein 1; PD-L1: Programmed death ligand 1; PFS: Progression-free survival; TC: Tumor cell; TCGA: The Cancer Genome Atlas; TMB: Tumor mutation burden
\end{abstract}

\section{Acknowledgements}

No additional acknowledgements other than those listed as authors.

\section{Funding}

There were no sources of funding for this review.

\section{Availability of data and materials}

Not applicable

\section{Authors' contributions}

Both authors contributed equally to the preparation of this review manuscript. Both authors read and approved the final manuscript.

\section{Authors' information \\ DHA is currently a Postdoctoral Clinical Fellow in Hematology and Oncology at Columbia University Medical Center with a research interest in genitourinary oncology and the identification and application of novel immunotherapies. CGD is a Professor of Medicine at Columbia University Medical Center where he serves as the Director of the Genitourinary Oncology Program. He has been senior author and principal investigator on numerous clinical trials in the identification of novel immune checkpoint molecules and the clinical development of multiple immune checkpoint inhibitors.}

\section{Ethics approval and consent to participate}

Not applicable

\section{Consent for publication}

Not applicable

\section{Competing interests}

DH. Aggen has no competing interests to report. CGD has stock or ownership in Compugen, Potenza Therapeutics, and Tizona Therapeutics. He has served in an advisory role or received research funding from Bristol-Myers Squibb, Compugen, Dendreon, Merck, Genentech, Eli Lilly, NexImmune, ImmuneXcite, Potenza Therapeutics, Tizona Therapeutics, Janssen Oncology (Inst), Regeneron and AstraZeneca/Medlmmune.

\section{Publisher's Note}

Springer Nature remains neutral with regard to jurisdictional claims in published maps and institutional affiliations.

Received: 17 July 2017 Accepted: 27 October 2017

Published online: 21 November 2017

\section{References}

1. Surveillance, Epidemiology, and End Results Program. SEER stat fact sheets: kidney and renal pelvis cancer. Bethesda: National Cancer Institute; 2017. at http://seer.cancer.gov/statfacts/html/urinb.html.

2. Siegel RL, Miller KD, Jemal A. Cancer statistics, 2017. CA Cancer J Clin. 2017; 67:7-30.

3. Bellmunt J, Orsola A, Leow JJ, et al. Bladder cancer: ESMO practice guidelines for diagnosis, treatment and follow-up. Ann Oncol. 2014;25(Suppl 3):iii40-8.

4. Milowsky MI, Rumble RB, Booth CM, et al. Guideline on muscle-invasive and metastatic bladder cancer (European Association of Urology guideline): American Society of Clinical Oncology clinical practice guideline endorsement. J Clin Oncol. 2016;34:1945-52.

5. Ghasemzadeh A, Bivalacqua TJ, Hahn NM, Drake CG. New strategies in bladder cancer: a second coming for immunotherapy. Clin Cancer Res. 2016;22:793-801.

6. Ning YM, Suzman D, Maher VE, et al. FDA Approval Summary: Atezolizumab for the Treatment of Patients with Progressive Advanced Urothelial Carcinoma after Platinum-Containing Chemotherapy. Oncologist. 2017;22(6):743-9.

7. Powles T, Eder JP, Fine GD, et al. MPDL3280A (anti-PD-L1) treatment leads to clinical activity in metastatic bladder cancer. Nature. 2014;515:558-62.

8. Rosenberg JE, Hoffman-Censits J, Powles T, et al. Atezolizumab in patients with locally advanced and metastatic urothelial carcinoma who have progressed following treatment with platinum-based chemotherapy: a single-arm, multicentre, phase 2 trial. Lancet. 2016;387:1909-20.

9. Hirsch FR, McElhinny A, Stanforth D, et al. PD-L1 Immunohistochemistry assays for lung cancer: results from phase 1 of the blueprint PD-L1 IHC assay comparison project. J Thorac Oncol. 2017;12:208-22.

10. Balar AV, Galsky MD, Rosenberg JE, et al. Atezolizumab as first-line treatment in cisplatin-ineligible patients with locally advanced and metastatic urothelial carcinoma: a single-arm, multicentre, phase 2 trial. Lancet. 2017; 389:67-76.

11. Sharma P, Retz M, Siefker-Radtke A, et al. Nivolumab in metastatic urothelial carcinoma after platinum therapy (CheckMate 275): a multicentre, singlearm, phase 2 trial. Lancet Oncol. 2017:18:312-22.

12. Raggi $D$, Miceli R, Sonpavde $G$, et al. Second-line single-agent versus doublet chemotherapy as salvage therapy for metastatic urothelial cancer: a systematic review and meta-analysis. Ann Oncol. 2016;27:49-61.

13. Bellmunt J, de Wit R, Vaughn DJ, et al. Pembrolizumab as second-line therapy for advanced Urothelial carcinoma. N Engl J Med. 2017;376:1015-26.

14. Herbst RS, Baas P, Kim DW, et al. Pembrolizumab versus docetaxel for previously treated, PD-L1-positive, advanced non-small-cell lung cancer (KEYNOTE-010): a randomised controlled trial. Lancet. 2016;387:1540-50.

15. Motzer RJ, Escudier B, McDermott DF, et al. Nivolumab versus Everolimus in advanced renal-cell carcinoma. N Engl J Med. 2015;373:1803-13.

16. Balar A, Bellmunt J, O'Donnell PH, et al. Pembrolizumab (pembro) as firstline therapy for advanced/unresectable or metastatic urothelial cancer: preliminary results from the phase 2 KEYNOTE-052 study. Ann Oncol. 2016; 27:LBA32_PR-LBA_PR.

17. O'Donnell PH, Grivas P, Balar AV, et al. Biomarker findings and mature clinical results from KEYNOTE-052: first-line pembrolizumab (pembro) in cisplatinineligible advanced urothelial cancer (UC). J Clin Oncol. 2017;35:4502.

18. Ayers M, Lunceford J, Nebozhyn M, et al. IFN-gamma-related mRNA profile predicts clinical response to PD-1 blockade. J Clin Invest. 2017:127(8):2930-40.

19. Massard C, Gordon MS, Sharma S, et al. Safety and efficacy of Durvalumab (MEDI4736), an anti-programmed cell death Ligand-1 immune checkpoint inhibitor, in patients with advanced Urothelial bladder cancer. J Clin Oncol. 2016;34:3119-25.

20. Drake CG, Bivalacqua TJ, Hahn NM. Programmed cell death Ligand-1 blockade in Urothelial bladder cancer: to select or not to select. J Clin Oncol. 2016:34:3115-6.

21. Hahn NM, Powles T, Massard C, et al. Updated efficacy and tolerability of durvalumab in locally advanced or metastatic urothelial carcinoma (UC). J Clin Oncol. 2017;35:4525. 
22. Apolo AB, Infante JR, Balmanoukian A, et al. Avelumab, an anti-programmed death-Ligand 1 antibody, in patients with refractory metastatic Urothelial carcinoma: results from a multicenter, phase Ib study. J Clin Oncol. 2017; 2017:JCO2016716795.

23. Patel MR, Ellerton JA, Infante JR, et al. Avelumab in patients with metastatic urothelial carcinoma: pooled results from two cohorts of the phase $1 \mathrm{~b}$ JAVELIN solid tumor trial. J Clin Oncol. 2017;35:330.

24. Larkin J, Chiarion-Sileni V, Gonzalez R, et al. Combined Nivolumab and Ipilimumab or Monotherapy in untreated melanoma. N Engl J Med. 2015; 373:23-34.

25. Hellmann MD, Rizvi NA, Goldman JW, et al. Nivolumab plus ipilimumab as first-line treatment for advanced non-small-cell lung cancer (CheckMate 012): results of an open-label, phase 1, multicohort study. Lancet Oncol. 2017;18:31-41

26. Sharma PCM, Calvo E, et al. Efficacy and safety of nivolumab plus ipilimumab in previously treated metastatic urothelial carcinoma: first results from the phase I/II CheckMate 032 study. J Clin Oncol. 2016;34(15 suppl): 2016. 2016 SITC Annual Meeting; National Harbor, MD

27. Callahan MK, Kania BE, lyer $\mathrm{G}$, et al. Evaluation of the clinical activity of ipilimumab (IPI) plus nivolumab (NIVO) in patients (pts) with NIVO-refractory metastatic urothelial cancer (UC). J Clin Oncol. 2017;35:384

28. Powles T, Galsky MD, Castellano D, et al. A phase 3 study of first-line durvalumab (MED|4736) \pm tremelimumab versus standard of care (SoC) chemotherapy (CT) in patients (pts) with unresectable stage IV urothelial bladder cancer (UBC): DANUBE. J Clin Oncol. 2016;34: TPS4574-TPS

29. Powles T, Gschwend JE, Loriot Y, et al. Phase 3 KEYNOTE-361 trial: Pembrolizumab (pembro) with or without chemotherapy versus chemotherapy alone in advanced urothelial cancer. J Clin Oncol. 2017;35: TPS4590-TPS

30. Smith DC, Gajewski T, Hamid O, et al. Epacadostat plus pembrolizumab in patients with advanced urothelial carcinoma: preliminary phase $\mathrm{I} / \mathrm{I}$ results of ECHO-202/KEYNOTE-037. J Clin Oncol. 2017;35:4503.

31. Rachel E, Sanborn MJP, Harriet M, Kluger, Callahan MK, Weise AM, Lutzky J, Yellin MJ, Rawls T, Vitale L, Halim A, Keler T, Davis T, Naiyer A, Rizvi. Clinical results with combination of anti-CD27 agonist antibody, varlilumab, with anti-PD1 antibody nivolumab in advanced cancer patients. Chicago: ASCO Annual Meeting 2017; 2017.

32. Infante JR, Ahlers CM, Hodi FS, et al. ENGAGE-1: a first in human study of the OX40 agonist GSK3174998 alone and in combination with pembrolizumab in patients with advanced solid tumors. J Clin Oncol. 2016;34:TPS3107-TPS.

33. Wainberg ZA, Eisenberg PD, Sachdev JC, et al. Phase 1/2a study of double immune suppression blockade by combining a CSF1R inhibitor (pexidartinib/ PLX3397) with an anti PD-1 antibody (pembrolizumab) to treat advanced melanoma and other solid tumors. J Clin Oncol. 2016;34:TPS465-TPS.

34. Apolo AB, Mortazavi A, Stein MN, et al. A phase I study of cabozantinib plus nivolumab (CaboNivo) and cabonivo plus ipilimumab (CaboNivolpi) in patients (pts) with refractory metastatic (m) urothelial carcinoma (UC) and other genitourinary (GU) tumors. J Clin Oncol. 2017;35:4562.

35. Kamat AM, Bellmunt J, Choueiri TK, et al. KEYNOTE-057: phase 2 study of pembrolizumab for patients (pts) with bacillus Calmette Guerin (BCG)unresponsive, high-risk non-muscle-invasive bladder cancer (NMIBC). J Clin Oncol. 2016;34:TPS4576-TPS.

36. Singh P, Catherine T, Lerner SP, et al. S1605: phase II trial of atezolizumab in BCG-unresponsive non-muscle invasive bladder cancer. J Clin Oncol. 2017; 35:TPS4591-TPS.

37. Boorjian SA, Sheinin Y, Crispen PL, et al. T-cell coregulatory molecule expression in urothelial cell carcinoma: clinicopathologic correlations and association with survival. Clin Cancer Res. 2008;14:4800-8.

38. Faraj SF, Munari E, Guner G, et al. Assessment of tumoral PD-L1 expression and intratumoral CD8+ T cells in urothelial carcinoma. Urology. 2015;85:703 e1-6.

39. Inman BA, Sebo TJ, Frigola $X$, et al. PD-L1 (B7-H1) expression by urothelial carcinoma of the bladder and BCG-induced granulomata: associations with localized stage progression. Cancer. 2007;109:1499-505.

40. Grigg C, Rizvi NA. PD-L1 biomarker testing for non-small cell lung cancer: truth or fiction? J Immunother Cancer. 2016;4:48.

41. Rimm DL, Han G, Taube JM, et al. A prospective, multi-institutional, pathologist-based assessment of 4 Immunohistochemistry assays for PD-L1 expression in non-small cell lung cancer. JAMA Oncol. 2017; 3(8):1051-58.
42. McLaughlin J, Han G, Schalper KA, et al. Quantitative assessment of the heterogeneity of PD-L1 expression in non-small-cell lung cancer. JAMA Oncol. 2016;2:46-54.

43. The Cancer Genome Atlas Research N. Comprehensive molecular characterization of urothelial bladder carcinoma. Nature. 2014;507:315-22.

44. Snyder A, Makarov V, Merghoub T, et al. Genetic basis for clinical response to CTLA-4 blockade in melanoma. N Engl J Med. 2014;371:2189-99.

45. Rizvi NA, Hellmann MD, Snyder A, et al. Cancer immunology. Mutational landscape determines sensitivity to PD-1 blockade in non-small cell lung cancer. Science. 2015;348:124-8.

46. Schumacher TN, Schreiber RD. Neoantigens in cancer immunotherapy. Science. 2015;348:69-74.

47. Rajasagi M, Shukla SA, Fritsch EF, et al. Systematic identification of personal tumor-specific neoantigens in chronic lymphocytic leukemia. Blood. 2014; 124:453-62.

48. McGranahan N, Furness AJ, Rosenthal R, et al. Clonal neoantigens elicit T cell immunoreactivity and sensitivity to immune checkpoint blockade. Science. 2016;351:1463-9.

49. Socinski M, Creelan B, Horn L, et al. NSCLC, metastaticCheckMate 026: a phase 3 trial of nivolumab vs investigator's choice (IC) of platinum-based doublet chemotherapy (PT-DC) as first-line therapy for stage iv/recurrent programmed death ligand 1 (PD-L1)-positive NSCLC. Ann Oncol. 2016;27: LBA7_PR-LBA_PR.

50. Carbone DP, Reck M, Paz-Ares L, et al. First-line Nivolumab in stage IV or recurrent non-small-cell lung cancer. N Engl J Med. 2017;376:2415-26.

51. Peters S. Impact of tumor mutation burden on the efficacy of firstline nivolumab in stage iv or recurrent non-small cell lung cancer: an exploratory analysis of CheckMate 026. Washington: American Association for Cancer Research Annual Meeting; 2017.

52. Hellmann MD, Sanchez-Vega F, La K, et al. Molecular determinants of response and resistance to anti-PD-(L)1 blockade in patients with NSCLC profiled with targeted next-generation sequencing (NGS). J Clin Oncol. 2017:35:9015.

53. Colli LM, Machiela MJ, Myers TA, Jessop L, Yu K, Chanock SJ. Burden of nonsynonymous mutations among TCGA cancers and candidate immune checkpoint inhibitor responses. Cancer Res. 2016;76:3767-72.

54. Mehnert JM, Panda A, Zhong $\mathrm{H}$, et al. Immune activation and response to pembrolizumab in POLE-mutant endometrial cancer. J Clin Invest. 2016:126:2334-40

55. Le DT, Uram JN, Wang H, et al. PD-1 blockade in tumors with mismatchrepair deficiency. N Engl J Med. 2015;372:2509-20.

56. Le DT, Uram JN, Wang H, et al. PD-1 blockade in mismatch repair deficient non-colorectal gastrointestinal cancers. J Clin Oncol. 2016;34:195.

57. Diaz LA, Le DT, Yoshino T, et al. KEYNOTE-177: randomized phase III study of pembrolizumab versus investigator-choice chemotherapy for mismatch repair-deficient or microsatellite instability-high metastatic colorectal carcinoma. J Clin Oncol. 2017;35:TPS815-TPS.

58. Skoulidis F, Hellmann MD, Awad MM, et al. STK11/LKB1 co-mutations to predict for de novo resistance to PD-1/PD-L1 axis blockade in KRAS-mutant lung adenocarcinoma. J Clin Oncol. 2017:35:9016.

59. Masucci GV, Cesano A, Hawtin R, et al. Validation of biomarkers to predict response to immunotherapy in cancer: volume I - pre-analytical and analytical validation. J Immunother Cancer. 2016;4:76.

60. Dobbin KK, Cesano A, Alvarez J, et al. Validation of biomarkers to predict response to immunotherapy in cancer: volume $\|$ - clinical validation and regulatory considerations. J Immunother Cancer. 2016:4:77.

61. Sweis RF, Galsky MD. Emerging role of immunotherapy in urothelial carcinoma-Immunobiology/biomarkers. Urol Oncol. 2016:34:556-65.

62. Cristescu RMR, Ayers M, Albright A, Murphy E, Yearley J, Sher X, Liu X, et al. Mutational load $(\mathrm{ML})$ and T-cell-inflamed microenvironment as preditors of response to pembrolizumab. Orlando: ASCO-SITC Clinical ImmunoOncology Symposium; 2017

63. Haddad Rl, Seiwert TY, Chow LQM, et al. Genomic determinants of response to pembrolizumab in head and neck squamous cell carcinoma (HNSCC). J Clin Oncol. 2017:35:6009.

64. Snyder A, Nathanson T, Funt SA, et al. Contribution of systemic and somatic factors to clinical response and resistance to PD-L1 blockade in urothelial cancer: an exploratory multi-omic analysis. PLoS Med. 2017;14:e1002309.

65. Chen $\mathrm{PL}$, Roh W, Reuben A, et al. Analysis of immune signatures in longitudinal tumor samples yields insight into biomarkers of response and mechanisms of resistance to immune checkpoint blockade. Cancer Discov. 2016;6:827-37. 
66. Gajewski TF. The next hurdle in cancer immunotherapy: overcoming the non-T-cell-inflamed tumor microenvironment. Semin Oncol. 2015; 42:663-71.

67. Sweis RF, Spranger $S$, Bao R, et al. Molecular drivers of the non-T-cellinflamed tumor microenvironment in Urothelial bladder cancer. Cancer Immunol Res. 2016;4:563-8.

68. Wallden B, Pekker I, Popa S, et al. Development and analytical performance of a molecular diagnostic for anti-PD1 response on the nCounter Dx analysis system. J Clin Oncol. 2016;34:3034.

69. Wallden BPI, Popa S, Dowidar N, Ngouenet C, Sullivan A, Danaher P, et al. Verification of the analytical performance of a molecular diagnostic for response to anti-PD1 therapy on the $\mathrm{nCounter}$ Dx analysis system. Orlando: 2017 ASCO-SITC Clinical Immuno-Oncology Symposium; 2017.

70. Diaz LA, Marabelle A, Delord J-P, et al. Pembrolizumab therapy for microsatellite instability high (MSI-H) colorectal cancer (CRC) and non-CRC. J Clin Oncol. 2017;35:3071.

71. Shah MA, Bennouna J, Shen $L$, et al. Pembrolizumab for previously treated metastatic adenocarcinoma or squamous cell carcinoma of the esophagus: phase 2 KEYNOTE-180 study. J Clin Oncol. 2016;34:TPS4139-TPS.

72. Doi T, Bennouna J, Shen L, et al. KEYNOTE-181: phase 3, open-label study of second-line pembrolizumab vs single-agent chemotherapy in patients with advanced/metastatic esophageal adenocarcinoma. J Clin Oncol. 2016;34: TPS4140-TPS.

Submit your next manuscript to BioMed Central and we will help you at every step:

- We accept pre-submission inquiries

- Our selector tool helps you to find the most relevant journal

- We provide round the clock customer support

- Convenient online submission

- Thorough peer review

- Inclusion in PubMed and all major indexing services

- Maximum visibility for your research

Submit your manuscript at www.biomedcentral.com/submit 